\title{
DISTRIBUTION OF PARTIAL SUMS OF THE TAYLOR DEVELOPMENT OF RATIONAL FUNCTIONS
}

\author{
V. NESTORIDIS
}

\begin{abstract}
Let $f$ be a rational function regular at 0 , which is not a polynomial; let $S_{N}(z), N=0,1,2, \ldots, z \in \mathbb{C}$, denote the partial sums of the Taylor development of $f$. We investigate the angular distribution of the sequence $S_{N}(z), N=0,1,2, \ldots$, around $f(z)$. We show that for all $z$ in the plane, except a denumerable union of straight lines passing through 0 , this angular distribution exists and is uniform.
\end{abstract}

Let $\sum_{0}^{\infty} a_{n} z^{n}, a_{n} \in \mathbb{C}$, be a power series which is $(C, 1)$ summable at every point $z$ of a set $E \subset\{z \in \mathbb{C}:|z|=1\}=T$. Let $\sigma(z) \in \mathbb{C}, z \in E$, be the $(C, 1)$ sum of $\sum_{0}^{\infty} a_{n} z^{n}$. For each fixed $z$ in $E$, we denote by $L(z)$ the set of limit points in $C \cup\{\infty\}$ of the sequence $S_{N}(z)=\sum_{0}^{N} a_{n} z^{n}, N=0,1, \ldots$

In 1941 Marcinkiewicz and Zygmund [8; 13, vol. II, p. 178] proved the following.

Theorem. Under the above assumptions, for almost all $z$ in $E$, the set $L(z)$ has circular structure with center $\sigma(z)$.

This means that, for almost all $z$ in $E$ and for every $w$ in $L(z)$, every point $w^{\prime}$ of the form $w^{\prime}=\sigma(z)+(w-\sigma(z)) e^{i \theta}, \theta \in \mathbf{R}$, also belongs to $L(z)$.

A. Zygmund asked whether the "angular distribution of $L(z)$ around $\sigma(z)$ " is uniform (see $[9,12]$ ); sometimes this question is called Rogosinski's problem. J.-P. Kahane in 1983 [2] introduced a large class of upper densities related to the limit points of a Taylor series; with respect to each one of these upper densities and for almost all $z$ in $E$, the distribution of $L(z)$ is invariant under rotations with center $\sigma(z)$.

S. K. Pichorides introduced the subject in Crete; in [3, 4, 10 and 5] one starts with geometric assumptions on the partial sums or on their limit points and concludes with analytic characterizations for the power series. In all these cases one can verify that the angular distribution is uniform.

The present paper deals with the problem of the angular distribution in the case where $\sum_{0}^{\infty} a_{n} z^{n}$ is the Taylor development of a rational function $f$ regular at 0 . A rational function is regular at 0 , if 0 is not a pole for this function. First we suppose that for some $z_{1},\left|z_{1}\right|=1$ the series is $(C, 1)$ summable but not convergent; it turns out that the same holds for all $z,|z|=1$, except the poles of $f$. Under these assumptions, we show that for all $z$ in the unit circle $T$, with a

Received by the editors August 24, 1993 and, in revised form, March 8, 1994.

1991 Mathematics Subject Classification. Primary 30B10, 28D05. 
denumerable set of exceptions, the limit set $L(z)$ has circular structure with center the $(C, 1)$ sum $\sigma(z)=f(z)$. This makes precise the "almost everywhere" statement in the theorem of Marcinkiewicz and Zygmund. The set of exceptions is related to the poles of $f$ in $T$. More precisely, if $e^{i 2 \pi \theta_{1}}, \ldots, e^{i 2 \pi \theta_{M}}$ are the poles of $f$ in $T$, the set of exceptions is contained in $e^{i 2 \pi V}$, where $V=\left\langle 1, \theta_{1}, \ldots, \theta_{M}\right\rangle_{Q}$ is the vector space over the field of rationals $Q$ generated by $1, \theta_{1}, \ldots, \theta_{M}$. The simple example $1 /(1-z)=\sum_{0}^{\infty} z^{n}$ shows that indeed we have an infinite denumerable set of exceptions. The proof of the above result is based on Kronecker's Theorem in Diophantine Approximation. Further, using Weyl's Uniform Distribution Theorem, we show, that under the above assumptions, for all $z$ in $T$ with a denumerable set of exceptions, the following holds: the sequence $S_{N}(z), N=0,1,2, \ldots$, is uniformly distributed with respect to a compactly supported in $\mathbb{C}$ probability measure $\mu_{z}$ which is invariant under rotations with center $f(z)$ and satisfies $\mu_{z}(\{f(z)\})=0$; it follows that the angular distribution of the sequence $S_{N}(z), N=0,1,2, \ldots$, around the $(C, 1)$ sum $\sigma(z)=f(z)$ exists and is uniform, for almost all $z$. It is worth noticing that, for rational functions, the weak assumption of $(C, 1)$ summability of the partial sums implies the existence of a distribution compactly supported in $C$; the converse trivially holds.

The above results, valid in the $(C, 1)$ summable case of rational functions, are used in order to prove our main result, where there is no assumption about $(C, 1)$ summability. This states the following.

Theorem. Let $f$ be a rational function regular at 0 , which is not a polynomial. Let $S_{N}(z), N=0,1,2, \ldots, z \in \mathbb{C}$, be the partial sums of the Taylor development of $f$. Then, there is a set $\Omega \subset \mathbb{C}$, which is a denumerable union of straight lines passing through 0 , such that the following holds:

For every $z$ in $\mathbb{C} \backslash \Omega$, the angular distribution of the sequence $S_{N}(z), N=$ $0,1,2, \ldots$, around $f(z)$ exists and is uniform.

The organization of the paper is as follows. Section 1 contains the characterization of rational functions with a nonconvergent $(C, 1)$ summable Taylor development. Section 2 deals with the distribution of the partial sums in the $(C, 1)$ summable case of rational functions. Section 3 contains the main result about the angular distribution without any assumptions about $(C, 1)$ summability.

Acknowledgment. The author wishes to express his gratitude to I. Deliyanni, C. Gryllakis, J.-P. Kahane, E. S. Katsoprinakis, S. Papadopoulou and S. K. Pichorides for helpful discussions and comments.

\section{Preliminaries}

We start with the following definition.

Definition 1. Let $A$ denote the set of rational functions $f$ with the following properties.

(i) $f$ is regular at 0 and therefore, $f$ has a Taylor development $\sum_{0}^{\infty} a_{n} z^{n}$.

(ii) There exists a point $z_{1},\left|z_{1}\right|=1$, such that the series $\sum_{0}^{\infty} a_{n} z_{1}^{n}$ is $(C, 1)$ summable to a finite sum, but is not convergent.

In this section we describe completely all elements of the set $A$. We need the following lemmas. 
Lemma 2. Let $M$ be a natural number, $M \geq 1$, let $c_{1}, \ldots, c_{M}$ be complex numbers and let $\varrho_{1}, \ldots, \varrho_{M}$ be complex numbers of absolute value one $\left(\left|\varrho_{j}\right|=1\right.$ for all $j=1, \ldots, M)$. We assume that $\varrho_{1}, \ldots, \varrho_{M}$ are distinct. We suppose that the sequence $\delta_{n}=\sum_{j=1}^{M} c_{j} \varrho_{j}^{n}, n=0,1, \ldots$, converges to zero. Then $c_{1}=\cdots=c_{M}=0$.

Proof. By a direct computation we have

$$
\begin{aligned}
\sum_{m=1}^{N}\left|\delta_{m}\right|^{2} & =\sum_{m=1}^{N} \delta_{m} \bar{\delta}_{m}=\sum_{m=1}^{N}\left(\sum_{k=1}^{M} c_{k} \varrho_{k}^{m}\right)\left(\sum_{\lambda=1}^{M} \bar{c}_{\lambda} \bar{\varrho}_{\lambda}^{m}\right)=\sum_{k, \lambda=1}^{M} c_{k} \bar{c}_{\lambda} \cdot \sum_{m=1}^{N}\left(\varrho_{k} \bar{\varrho}_{\lambda}\right)^{m} \\
& =N \sum_{k=1}^{M}\left|c_{k}\right|^{2}+\sum_{\substack{k, \lambda=1 \\
k \neq \lambda}} c_{k} \bar{c}_{\lambda} \varrho_{k} \bar{\varrho}_{k}\left[1-\left(\varrho_{k} \bar{\varrho}_{\lambda}\right)^{N}\right] /\left(1-\varrho_{k} \bar{\varrho}_{\lambda}\right) .
\end{aligned}
$$

Obviously $\left|1-\left(\varrho_{k} \bar{\varrho}_{k}\right)^{N}\right| \leq 2$ and $\left[1-\left(\varrho_{k} \bar{\varrho}_{\lambda}\right)^{N}\right] / N \rightarrow 0$, as $N \rightarrow+\infty$. It follows that $(1 / N) \sum_{m=1}^{N}\left|\delta_{m}\right|^{2} \rightarrow \sum_{k=1}^{M}\left|c_{k}\right|^{2}$, as $N \rightarrow+\infty$. Since $\delta_{n} \rightarrow 0$, as $n \rightarrow+\infty$, we conclude $\sum_{k=1}^{M}\left|c_{k}\right|^{2}=0$; this gives $c_{1}=\cdots=c_{M}=0$. Q.E.D.

There are several proofs of Lemma 2 (see $[6,11]$ ); the proof given here is due to N. Alikakos.

Lemma 3. Let $\sum_{n=0}^{\infty} \gamma_{n}$ be a complex series, which is $(C, 1)$ summable to a finite sum $\sigma$. Then $\gamma_{n}=o(n)$, as $n \rightarrow+\infty$.

Proof. Let $S_{N}=\sum_{n=0}^{N} \gamma_{n}$ and $\sigma_{N}=\left(S_{0}+\cdots+S_{N}\right) /(N+1) \rightarrow \sigma \in C$. Then we have $(N+1) \sigma_{N}-N \sigma_{N-1}=S_{N}$ and $\gamma_{N}=S_{N}-S_{N-1}=(N+1) \sigma_{N}-2 N \sigma_{N-1}+$ $(N-1) \cdot \sigma_{N-2}$. It follows that $\gamma_{N} / N \rightarrow 0$, as $N \rightarrow+\infty$. Q.E.D.

Lemma 3 above is well known (see [13]); we included its short proof, for the purpose of completeness.

Proposition 4. Let $f$ be a regular at 0 rational function and let $\sum_{0}^{\infty} a_{n} z^{n}$ be the Taylor development of $f$. We suppose that there exists $z_{1},\left|z_{1}\right|=1$, such that the series $\sum_{0}^{\infty} a_{n} z_{1}^{n}$ is $(C, 1)$ summable to a finite sum. Then we have the following.

(a) $f$ is regular in the open unit disk $D=\{z \in \mathbb{C}:|z|<1\}$.

(b) If $f$ has poles on the unit circle $T$, then these poles are simple.

(c) $f=\omega+g$ with $g$ a rational function regular in a neighbourhood of the closed unit disk $\bar{D}$ and $\omega(z)=\sum_{j=1}^{M} A_{j} /\left(1-\varrho_{j} z\right), M \geq 1, A_{j} \in \mathbb{C}, \varrho_{j}=e^{i 2 \pi \theta_{j}}$, $0<\theta_{j} \leq 1$ for all $j=1, \ldots, M$ and $\varrho_{1}, \ldots, \varrho_{M}$ are distinct.

Proof. Since $\sum_{0}^{\infty} a_{n} z_{1}^{n}\left(\left|z_{1}\right|=1\right)$ is $(C, 1)$ summable to a finite sum, according to Lemma 3, we have $a_{n} / n \rightarrow 0$, as $n \rightarrow+\infty$. It follows that the radius of convergence of $\sum_{0}^{\infty} a_{n} z^{n}$ is greater than or equal to 1 . On the other hand, this radius of convergence is the distance of 0 to the nearest pole of $f$. We conclude that $f$ is regular in the open unit disk. This gives part (a).

Expanding $f$ to simple fractions, we obtain rational functions $\omega$ and $g$, such that $f=\omega+g, g$ is regular in a neighbourhood of $\bar{D}$ and $\omega$. If $g$ has any poles in $C \cup\{\infty\}$, they belong to the unit circle $T$. We may also assume $\omega(\infty)=0$.

Suppose $\omega$ has distinct poles $\xi_{1}, \ldots, \xi_{M}$ with multiplicities $\nu_{1}, \ldots, \nu_{M}$, respectively. We have $\left|\xi_{j}\right|=1$ and $\nu_{j} \geq 1$ for $j=1, \ldots, M$. We shall 
prove that $\nu_{j}=1$ for all $j=1, \ldots, M$. Suppose on the contrary that $\max \left\{\nu_{1}, \ldots, \nu_{M}\right\}=m \geq 2$, to arrive at a contradiction.

Consider the nonvoid set $B=\left\{k \in\{1, \ldots, M\}: \nu_{k}=m\right\}$; then $\omega$ has the form

$$
\omega(z)=\sum_{k \in B} \Gamma_{k} /\left(1-\bar{\xi}_{k} z\right)^{m}+\sum_{s=1}^{m-1} \sum_{j=1}^{M} \Delta_{j, s} /\left(1-\bar{\xi}_{j} z\right)^{s},
$$

where $\Gamma_{k} \neq 0$, for all $k \in B$.

The Taylor development $\sum_{0}^{\infty} a_{n} z^{n}$ of $f$ is the sum of those of $\omega$ and $g$. Since the Taylor development of $g$ is convergent at $z_{1}$, and $\sum_{0}^{\infty} a_{n} z_{1}^{n}$ is $(C, 1)$ summable, we see that the series $\omega(z)=\sum_{0}^{\infty} \beta_{n} z^{n}$ is $(C, 1)$ summable at $z=z_{1}$. According to Lemma 3 , there holds $\beta_{n} / n \rightarrow 0$, as $n \rightarrow+\infty$.

In order to compute $\beta_{n}$, we need the expansion $1 /(1-\xi z)^{l}=\sum_{0}^{\infty}\left(\begin{array}{c}n+l-1 \\ l-1\end{array}\right) \xi^{n} z^{n}$ $=\sum_{0}^{\infty}(n+l-1) \cdots(n+1) /(l-1) ! \xi^{n} z^{n}, l=1,2, \ldots$.

It follows that

$$
\beta_{n}=(n+m-1) \cdots(n+1) /(m-1) !\left[\sum_{k \in B} \Gamma_{k} \bar{\xi}_{k}^{n}+O(1 / n)\right] .
$$

Since $m \geq 2$ we see that $(n+m-1) \cdots(n+1) / n \cdot(m-1)$ ! is bounded below away from zero; we also have $\beta_{n} / n \rightarrow 0$, as $n \rightarrow+\infty$. It follows that $\sum_{k \in B} \Gamma_{k} \bar{\xi}_{k}^{n} \rightarrow 0$, as $n \rightarrow+\infty$. According to Lemma 2, we have $\Gamma_{k}=0$, for all $k \in B$; this gives a contradiction and therefore, $\max \left\{\nu_{1}, \ldots, \nu_{M}\right\}=m=1$ and $B=\{1, \ldots, M\}$. Thus, every pole of $f$ in the unit circle is simple; this gives (b). It follows that $\omega(z)=\sum_{j=1}^{M} A_{j} /\left(1-\varrho_{j} z\right)$ with $M \geq 1, A_{j} \in \mathbb{C}, \varrho_{j}=$ $\bar{\xi}_{j}=e^{i 2 \pi \theta_{j}}, 0<\theta_{j} \leq 1$, and $\varrho_{1}, \ldots, \varrho_{M}$ are distinct. This gives (c) and completes the proof. Q.E.D.

One can easily see that the converse of Proposition 4 holds. More precisely, with the notation of Proposition 4, we suppose that (c) holds; then, one can easily verify that, for every $z$ in $T, z \neq \bar{\varrho}_{j}, j=1, \ldots, M$, the series $\sum_{n=0}^{\infty} a_{n} z^{n}$ is $(C, 1)$ summable to the finite sum $\sigma(z)=f(z)$.

This verification reduces to the simple case $1 /(1-z)=\sum_{0}^{\infty} z^{n}$; then $S_{N-1}(z)$ $=\left(1-z^{N}\right) /(1-z)$ for $z \neq 1$. It follows that $\sigma_{N}(z)=S_{0}(z)+\cdots+S_{N}(z) / N+1$ $=1 /(1-z)-[1 /(N+1)] z\left(1-z^{N+1}\right) /(1-z)^{2}$ and $\sigma_{N}(z) \rightarrow \sigma(z)=$ $1 /(1-z)$, for all $z,|z|=1, z \neq 1$.

Now, we describe the elements of the set $A$ introduced in Definition 1.

If in Proposition 4 we have $\omega \equiv 0$, then $f \equiv g$ is regular on $\bar{D}$ and the Taylor development $f(z)=\sum_{0}^{\infty} a_{n} z^{n}$ converges, for every $z$ in the unit circle $T$. If $\omega$ is not identically zero, then we have $\omega(z)=\sum_{j=1}^{M} A_{j} / 1-\varrho_{j} z, M \geq 1$, $A_{j} \neq 0,\left|\varrho_{j}\right|=1$ for all $j=1, \ldots, M$ and $\varrho_{1}, \ldots, \varrho_{M}$ are distinct. We have the Taylor development $\omega(z)=\sum_{n=0}^{\infty} \beta_{n} z^{n}$ with $\beta_{n}=\sum_{1}^{M} A_{j} \varrho_{j}^{n}$. According to Lemma $2, \beta_{n}$ cannot converge to zero. It follows that $\sum_{n=0}^{\infty} \beta_{n} z^{n}$ does not converge, for any $z$ in $T$. Since $g$ is regular on $\bar{D}$, its Taylor development is convergent to any $z$ in $T$. It follows that the Taylor development of $f=\omega+g$ is not convergent at any $z$ in $T$ (provided $\omega \equiv 0$ ). Thus, we have proved the following characterization of the elements of the set $A$ (see Definition 1). 
Proposition 5. A function $f$ belongs to the set $A$, if and only if, $f=\omega+g$ with $g$ a rational function regular in a neighbourhood of the closed unit disk $\bar{D}$ and $\omega(z)=\sum_{j=1}^{M} A_{j} /\left(1-\varrho_{j} z\right), M \geq 1, A_{j} \neq 0, \varrho_{j}=e^{i 2 \pi \theta_{j}}, 0<\theta_{j} \leq 1$, for all $j=$ $1, \ldots, M$ and $\varrho_{1}, \ldots, \varrho_{M}$ are distinct.

\section{Distribution of Partial SUMS}

Let $f=\omega+g$ be a function belonging to the set $A$ introduced in $\S 1$. According to Proposition $5, g$ is a rational function regular on $\bar{D}$ and $\omega(z)=$ $\sum_{j=1}^{M} A_{j} /\left(1-\varrho_{j} z\right)$ with $M \geq 1, A_{j} \neq 0, \varrho_{j}=e^{i 2 \pi \theta_{j}}, 0<\theta_{j} \leq 1$, for all $j=1, \ldots, M$ and $\varrho_{1}, \ldots, \varrho_{m}$ are distinct.

We denote by $S_{N}(z), \omega_{N}(z), g_{N}(z)$ the $N$ th partial sums of the Taylor developments of $f, \omega$ and $g$, respectively. Obviously $S_{N}(z)=g_{N}(z)+\omega_{N}(z)$ and, for every $z,|z|=1$, we have the convergence $g_{N}(z) \rightarrow g(z)$, as $N \rightarrow$ $+\infty$, because $g$ is regular in a neighbourhood of $\bar{D}$. By a direct computation we find $S_{N-1}(z)=g_{N-1}(z)+\omega(z)+\left\{\sum_{j=1}^{M}\left[A_{j} /\left(\varrho_{j} z-1\right)\right] \varrho_{j}^{N}\right\} z^{N}$. For every $z$ in the unit circle $T$, we have $g_{N-1}(z)+\omega(z) \rightarrow g(z)+\omega(z)=f(z)$, as $N \rightarrow+\infty$, and $f(z)$ is the $(C, 1)$ sum of the Taylor development of $f$. Therefore, it suffices to study the distribution of the sequence $\delta_{N}(z)=$ $\left\{\sum_{j=1}^{M}\left[A_{j} /\left(\varrho_{j} z-1\right)\right] \varrho_{j}^{N}\right\} z^{N}, N=1,2, \ldots$, and its angular distribution around 0 .

Lemma 6. Let $\delta_{N}(z, w)=\left\{\sum_{j=1}^{M}\left[A_{j} /\left(\varrho_{j} w-1\right)\right] \varrho_{j}^{N}\right\} z^{N}, N=1,2, \ldots$, where $M \geq 1, A_{j} \neq 0, \varrho_{j}=e^{i 2 \pi \theta_{j}}, 0<\theta_{j} \leq 1$, and $\varrho_{1}, \ldots, \varrho_{M}$ are distinct. The parameter $w$ is complex and different from $\bar{\varrho}_{j}, j=1, \ldots, M$. Let $V=$ $\left\langle 1, \theta_{1}, \ldots, \theta_{M}\right\rangle_{Q}$ be the vector space over the rationals generated by $1, \theta_{1}, \ldots$, $\theta_{M}$ and let $X=e^{i 2 \pi V}$, which is a denumerable subset of the unit circle $T$. We also denote by $E(z, w)$ the set of limit points in $\mathbb{C} \cup\{\infty\}$ of the sequence $\delta_{N}(z, w), N=1,2, \ldots$. Then, there is a finite set $B \subset \mathbb{C}$, such that the following hold.

(a) For every $z \in T \backslash X$ the set $E(z, w)$ is a compact subset of $\mathbb{C}$, it has a finite number of connected components and it has circular structure with center 0 .

(b) For every $z \in T \backslash X$ and every $w \in C \backslash B$, there is a probability measure $\lambda_{z, w}$ compactly supported in $\mathbb{C}$ such that

(i) The sequence $\delta_{N}(z, w), N=1,2, \ldots$, is uniformly distributed with respect to the measure $\lambda_{z, w}$.

(ii) The measure $\lambda_{z, w}$ is invariant under rotations with center 0 .

(iii) $\lambda_{z, w}(\{0\})=0$.

(iv) The sequence $\operatorname{card}\left\{N: 1 \leq N \leq P, \delta_{N}(z, w) \in \Phi\right\} / P, P=$ $1,2, \ldots$, converges and its limit is $\lambda_{z, w}(\Phi)=(\beta-\alpha) / 2 \pi$, where $\Phi=\left\{r e^{i t}: 0<r, \alpha<t<\beta\right\}, \alpha<\beta \leq \alpha+2 \pi$.

Proof. Let $1=\omega_{0}, \omega_{1}, \ldots, \omega_{s}, 0 \leq s \leq M$, be a $Q$-base of the vector space $V=\left\langle 1, \theta_{1}, \ldots, \theta_{M}\right\rangle_{Q}$. Therefore, there exist a natural number $m \geq 1$ and integer numbers $a_{j}, \beta_{k}^{j}, j=1, \ldots, M, 1 \leq k \leq s$, such that $\theta_{j}=$ $(1 / m)\left[a_{j} .1+\beta_{1}^{j} \omega_{1}+\cdots+\beta_{s}^{j} \omega_{s}\right]$, for all $j=1, \ldots, M$.

Fix $z=e^{i 2 \pi x}$ with $x \in \mathbf{R} \backslash V$; that is, $z \in T \backslash X$. Obviously the system $1, \omega_{1}, \ldots, \omega_{s}, x$ is $Q$-independent. Kronecker's Theorem [1] asserts that the 
sequence $\zeta_{\nu}=\left(e^{i 2 \pi \omega_{1} \nu}, \ldots, e^{i 2 \pi \omega_{s} \nu}, e^{i 2 \pi x \nu}\right), \nu=0,1,2, \ldots$, is dense in the torus $T^{S+1}$. Further, by Weyl's uniform distribution theorem [7], the sequence $\zeta_{\nu}, \nu=0,1, \ldots$, is uniformly distributed with respect to the normalized Lebesgue measure $\sigma_{s+1}$ on $T^{S+1}$.

For every fixed $v=0, \ldots, m-1$ and $z=e^{i 2 \pi x} \in T \backslash X$ we consider the sequence $\gamma_{\nu}^{v}(z, w)=\delta_{\nu m+v}(z, w)=\left\{\sum_{j=1}^{M}\left[A_{j} /\left(\varrho_{j} w-1\right)\right]\left(\varrho_{j} z\right)^{v}\left(e^{i 2 \pi \omega_{1} \nu}\right)^{\beta_{1}^{j}} \ldots\right.$ $\left.\left(e^{i 2 \pi \omega_{s} \nu}\right)^{\beta_{s}^{j}}\right\}\left(e^{i 2 \pi x \nu}\right)^{m}, \nu=0,1,2, \ldots$, where we use the fact that the exponents $\beta_{k}^{j}$ and $m$ are integer numbers. We denote by $E^{v}(z, w)$ the limit set of the sequence $\gamma_{\nu}^{v}(z, w), \nu=0,1, \ldots$. Obviously

$$
E(z, w)=\bigcup_{v=0}^{m-1} E^{v}(z, w) .
$$

We introduce the functions

$$
\begin{aligned}
g_{z, w}^{v}\left(z_{1}, \ldots, z_{s}, z_{0}\right) & =\left\{\sum_{j=1}^{M}\left[A_{j} /\left(\varrho_{j} w-1\right)\right]\left(\varrho_{j} z\right)^{v}\left(z_{1}^{\beta_{1}^{j}} \cdots z_{s}^{\beta_{s}^{j}}\right)\right\} . z_{0}^{m} \\
& \equiv h_{z, w}^{v}\left(z_{1}, \ldots, z_{s}\right) \cdot z_{0}^{m}
\end{aligned}
$$

which are continuous on the torus $T^{s+1}$ (see also [6 and 11]). Obviously $\gamma_{\nu}^{v}(z, w)=g_{z, w}^{v}\left(\zeta_{\nu}\right) \in g_{z, w}^{v}\left(T^{S+1}\right)$, for all $\nu=0,1,2, \ldots$. The set $g_{z, w}^{v}\left(T^{S+1}\right)$ is connected and compact; therefore, the limit set $E^{v}(z, w)$ satisfies $E^{v}(z, w)$ $\subset g_{z}^{v}, w\left(T^{S+1}\right)$. Since the sequence $\zeta_{\nu}=\left(e^{i 2 \pi \omega_{1} \nu}, \ldots, e^{i 2 \pi \omega_{s} \nu}, e^{i 2 \pi x \nu}\right), \nu=$ $0,1,2, \ldots$, is dense in $T^{S+1}$ and the function $g_{z}^{v}, w$ is continuous on $T^{S+1}$, we conclude that the sequence $\gamma_{\nu}^{v}(z, w)=g_{z, w}^{v}\left(\zeta_{\nu}\right), \nu=0,1,2, \ldots$, is dense in $g_{z, w}^{v}\left(T^{S+1}\right)$. There follow the equalities

$$
E^{v}(z, w)=g_{z, w}^{v}\left(T^{S+1}\right) \text { and } E(z, w)=\bigcup_{v=0}^{m-1} g_{z, w}^{v}\left(T^{S+1}\right) .
$$

In order to prove (i), it suffices to show that, for each $v=0, \ldots, m-1$, the compact and connected set $g_{z, w}^{v}\left(T^{S+1}\right) \subset \mathbb{C}$ has circular structure around 0 , where $g_{z, w}^{v}=h_{z, w}^{v}\left(z_{1}, \ldots, z_{s}\right) \cdot z_{0}^{m}$. Indeed, let $\theta \in \mathbf{R}$ and $\left(z_{1}, \ldots, z_{s}, z_{0}\right)$ $\in T^{S+1}$; we have $e^{i \theta}\left[g_{z, w}^{v}\left(z_{1}, \ldots, z_{s}, z_{0}\right)\right]=e^{i \theta}\left[h_{z, w}^{v}\left(z_{1}, \ldots, z_{s}\right) . z_{0}^{m}\right]$ $=h_{z, w}^{v}\left(z_{1}, \ldots, z_{s}\right) \cdot\left(e^{i \theta / m} z_{0}\right)^{m}=g_{z, w}^{v}\left(z_{1}, \ldots, z_{s}, e^{i \theta / m} z_{0}\right)$ and

$$
\left(z_{1}, \ldots, z_{s}, e^{i \theta / m} z_{0}\right) \in T^{S+1} \text {. }
$$

This completes the proof of (i).

To prove part (ii) we consider the measures $\lambda_{z, w}^{v}, z=e^{i 2 \pi x} \in T \backslash X, v=$ $0, \ldots, m-1$, defined as follows: $\lambda_{z, w}^{v}(\Delta)=\sigma_{S+1}\left[\left(g_{z, w}^{v}\right)^{-1}(\Delta)\right]$, for every Borel set $\Delta$ of $\mathbb{C}$; equivalently, $\int \varphi d \lambda_{z, w}^{v}=\int \varphi \circ g_{z}^{v}, w d \sigma_{S+1}$, for every continuous function $\varphi$ on $\mathbb{C}$. In other words $\lambda_{z, w}^{v}$ is the measure image by $g_{z, w}^{v}$ of the normalized Lebesgue measure $\sigma_{S+1}$ on $T^{S+1}$. It follows that $\lambda_{z, w}^{v}$ is a probability measure supported in the compact set $g_{z, w}^{v}\left(T^{S+1}\right)=E^{v}(z, w)$.

Since the sequence $\zeta_{\nu}=\left(e^{i 2 \pi \omega_{1} \nu}, \ldots, e^{i 2 \pi \omega_{s} \nu}, e^{i 2 \pi x \nu}\right), \nu=0,1,2, \ldots$, is uniformly distributed with respect to the measure $\sigma_{S+1}$ and $g_{z, w}^{v}$ is continuous on $T^{S+1}$, passing to the images by $g_{z, w}^{v}$, we have the following (see [7, p. 179]): 
The sequence $g_{z, w}^{v}\left(\zeta_{\nu}\right)=\gamma_{\nu}^{v}(z, w)=\delta_{\nu m+v}(z, w), \nu=0,1,2, \ldots$, is uniformly distributed with respect to the measure $\lambda_{z, w}^{v}$.

Claim. The measure $\lambda_{z, w}^{v}$ is invariant under rotations with center 0 .

Indeed, let $\Delta$ be a Borel subset of $\mathbb{C}$ and $\theta \in \mathbf{R}$; then using Fubini's theorem we have

$$
\begin{aligned}
\lambda_{z, w}^{v}\left(e^{i \theta} \Delta\right) & =\sigma_{S+1}\left[\left(g_{z, w}^{v}\right)^{-1}\left(e^{i \theta} \Delta\right)\right] \\
& =\sigma_{S+1}\left(\left\{z_{1}, \ldots, z_{s}, z_{0}\right) \in T^{S+1}: g_{z, w}^{v}\left(z_{1}, \ldots, z_{s}, z_{0}\right)\right. \\
& \left.\left.=h_{z, w}^{v}\left(z_{1}, \ldots, z_{s}\right) z_{0}^{m} \in e^{i \theta} \Delta\right\}\right) \\
& =\sigma_{S+1}\left(\left\{z_{1}, \ldots, z_{s}, z_{0}\right) \in T^{S+1}:\right. \\
& \left.\left.\quad h_{z, w}^{v}\left(z_{1}, \ldots, z_{s}\right) .\left(e^{-i \theta / m} z_{0}\right)^{m} \in \Delta\right\}\right) \\
& \left.=\sigma_{S+1}\left(\left\{z_{1}, \ldots, z_{s}, e^{i \theta / m} \tau\right) \in T^{S+1}: h_{z, w}^{v}\left(z_{1}, \ldots, z_{s}\right) \tau^{m} \in \Delta\right\}\right) \\
& =\int_{T^{S}} \sigma_{1}\left(\left\{e^{i \theta / m} \tau \in T: h_{z, w}^{v}\left(z_{1}, \ldots, z_{s}\right) . \tau^{m} \in \Delta\right\}\right) d \sigma_{s}\left(z_{1}, \ldots, z_{s}\right) .
\end{aligned}
$$

The invariance of the Lebesgue measure $\sigma_{1}$ on $T$ by rotations with center 0 gives

$$
\sigma_{1}\left(\left\{e^{i \theta / m} \tau: h_{z, w}^{v}\left(z_{1}, \ldots, z_{s}\right) . \tau^{m} \in \Delta\right\}\right)=\sigma_{1}\left(\left\{\tau: h_{z, w}^{v}\left(z_{1}, \ldots, z_{s}\right) . \tau^{m} \in \Delta\right\}\right) .
$$

Therefore,

$$
\lambda_{z, w}^{v}\left(e^{i \theta} \Delta\right)=\int_{T^{s}} \sigma_{1}\left(\left\{\tau \in T: h_{z, w}^{v}\left(z_{1}, \ldots, z_{s}\right) . \tau^{m} \in \Delta\right\}\right) d \sigma_{s}\left(z_{1}, \ldots, z_{s}\right) .
$$

Repeating the previous calculations with $\theta=0$, we see that this last integral is equal to $\lambda_{z, w}^{v}(\Delta)$. Therefore, we have $\lambda_{z, w}^{v}\left(e^{i \theta} \Delta\right)=\lambda_{z, w}^{v}(\Delta)$, which proves the claim.

Next we claim that, for every $w$ in $\mathbb{C}$, except a finite set $\Omega^{v}$, measure $\lambda_{z, w}^{v}$ satisfies $\lambda_{z, w}^{v}(\{0\})=0$, for all $z$ in $T \backslash X$.

Indeed, if $\lambda_{z, w}^{v}(\{0\})>0$ then $g_{z, w}^{v}=0$ on a subset of $T^{S+1}$ with strictly positive Lebesgue measure. We can multiply $g_{z, w}^{v}$ by a monomial to obtain a polynomial in $S+1$ variables. This polynomial must vanish identically. Therefore, all its coefficients must be equal to zero. A specific coefficient has the form

$$
\sum_{j \in \Gamma}\left[A_{j} /\left(\varrho_{j} w-1\right)\right]\left(\varrho_{j} z\right)^{v} \equiv C^{v}(w) . z^{v},
$$

where $\Gamma=\left\{j \in\{1, \ldots, M\}: \beta_{1}^{j}=\beta_{1}^{1}, \ldots, \beta_{s}^{j}=\beta_{s}^{1}\right\}, 1 \in \Gamma$.

Since $A_{j} \neq 0$, for all $j=1, \ldots, M$ and $\varrho_{1}, \ldots, \varrho_{M}$ are distinct, we see that $C^{v}(w)$ is a rational function of $w$ with pole at $\bar{\varrho}_{1}$; therefore, $C^{v}(w)$ is a nonidentically zero rational function. Its zero set is finite. We call $\Omega^{v}$ this set and we have the claim.

Let $z \in T \backslash X$ and $w \in C \backslash\left(\Omega^{v} \cup\left\{\bar{\varrho}_{1}, \ldots, \bar{\varrho}_{M}\right\}\right)$ for some $v=0, \ldots, m-1$. Since $\lambda_{z, w}^{v}$ is a probability measure, invariant by rotations with center 0 and $\lambda_{z, w}^{v}(\{0\})=0$, we have the following:

(i) For every closed half line $\varepsilon$ starting at 0 , we have $\lambda_{z, w}^{v}(\varepsilon)=0$.

(ii) If $\Phi=\left\{r e^{i t}: 0<r, \alpha<t<\beta\right\}, \alpha<\beta \leq \alpha+2 \pi$, then $\lambda_{z, w}^{v}(\Phi)=$ $(\beta-\alpha) / 2 \pi$ and $\lambda_{z, w}^{v}(\partial \Phi)=0$. 
Further, since $\lambda_{z, w}^{v}$ has compact support in $\mathbb{C}$, we can find a closed disk $D_{z, w}^{v}$ centered at 0 , containing in its interior the compact support of $\lambda_{z, w}^{v}$. We may restrict our attention in the compact set $D_{z, w}^{v}$. If $\Phi$ is as in (ii) above, then $\lambda_{z, w}^{v}\left(\Phi \cap D_{z, w}^{v}\right)=\lambda_{z, w}^{v}(\Phi)=(\beta-\alpha) / 2 \pi$ and $\lambda_{z, w}^{v}\left(\partial\left[\Phi \cap D_{z, w}^{v}\right]\right)=$ 0 . We also have the equivalence: $\gamma_{\nu}^{v}(z, w) \in \Phi \Leftrightarrow \gamma_{\nu}^{v}(z, w) \in \Phi \cap D_{z, w}^{v}$. Since the sequence $\gamma_{\nu}^{v}(z, w), \nu=0,1,2, \ldots$, is uniformly distributed with respect to $\lambda_{z, w}^{v}$, we conclude that (see $[7$, p. 175]) the sequence $\operatorname{card}\{\nu: 0 \leq$ $\left.\nu \leq N, \gamma_{\nu}^{v}(z, w) \in \Phi\right\} /(N+1), N=0,1,2, \ldots$, converges and its limit is $\lambda_{z, w}^{v}(\Phi)=(\beta-\alpha) / 2 \pi$.

Therefore, the angular distribution of the sequence $\gamma_{\nu}^{v}(z, w)=\delta_{\nu m+v}(z, w)$, $\nu=0,1,2, \ldots$, exists and is uniform.

Let $B=\Omega^{0} \cup \cdots \cup \Omega^{m-1} \cup\left\{\bar{\varrho}_{1}, \ldots, \bar{\varrho}_{M}\right\}$, which is a finite set. For every $z \in T \backslash X$ and $w \in \mathbb{C} \backslash B$ we consider the probability measure

$$
\lambda_{z, w}=(1 / m)\left(\lambda_{z, w}^{0}+\cdots+\lambda_{z, w}^{m-1}\right) .
$$

We easily obtain part (b) of the lemma. Q.E.D.

Since $S_{N-1}(z)=g_{N-1}(z)+\omega(z)+\delta_{N}(z, z)$ and $g_{N-1}(z)+\omega(z) \rightarrow g(z)+$ $\omega(z)=f(z)$, as $N \rightarrow \infty$, Lemma 6 implies obviously the following.

Theorem 7. Let $f=\omega+g$ be a rational function belonging to the set $A$; that is, $g$ is a rational function regular on $\bar{D}$ and $\omega(z)=\sum_{j=1}^{M} A_{j} /\left(1-\varrho_{j} z\right)$ with $M \geq 1, A_{j} \neq 0, \varrho_{j}=e^{i 2 \pi \theta_{j}}, 0<\theta_{j} \leq 1$, for all $j=1, \ldots, M$ and $\varrho_{1}, \ldots, \varrho_{M}$ are distinct.

Let $V=\left\langle 1, \theta_{1}, \ldots, \theta_{M}\right\rangle_{Q}$ be the vector space over the rationals generated by $1, \theta_{1}, \ldots, \theta_{M}$ and let $X=e^{i 2 \pi V}$.

Let $\sum_{0}^{\infty} a_{n} z^{n}$ be the Taylor development of $f$ and $S_{N}(z)=\sum_{0}^{N} a_{n} z^{n}$. We denote by $L(z)$ the set of limit points in $\mathbb{C} \cup\{\infty\}$ of the sequence $S_{N}(z)$, $N=0,1,2, \ldots$.

Then we have the following:

(a) For every $z$ in the unit circle $T$, except the denumerable set $X$, the set $L(z)$ is a compact subset of $\mathbb{C}$, it has a finite number of connected components and it has circular structure with center the $(C, 1)$ sum $\sigma(z)=f(z)$ of the series $\sum_{0}^{\infty} a_{n} z^{n}$. hold.

(b) There is a finite set $B$, such that, for every $z$ in $T \backslash(X \cup B)$ the following

(i) There exists a Borel probability measure $\mu_{z}$ with compact support in $\mathbb{C}$, such that the sequence $S_{N}(z), N=0,1,2, \ldots$, is uniformly distributed with respect to the measure $\mu_{z}$. series $\sum_{0}^{\infty} a_{n} z^{n}$.

(ii) $\mu_{z}(\{\sigma(z)\})=0$, where $\sigma(z)=f(z)$ is the $(C, 1)$ sum of the $f(z)$.

(iii) The measure $\mu_{z}$ is invariant under rotations with center $\sigma(z)=$

(iv) Let $\Phi$ be an open angle with vertex $\sigma(z)=f(z)$ and opening $\varphi$, $0<\varphi \leq 2 \pi$. Then the sequence $\operatorname{card}\left\{n: 0 \leq n \leq N, S_{n}(z) \in \Phi\right\} /(N+1)$, $N=0,1,2, \ldots$, is convergent and its limit is equal to $\mu_{z}(\Phi)=\varphi / 2 \pi$. Thus, the angular distribution of the sequence $S_{N}(z), N=0,1,2, \ldots$, around the $(C, 1)$ sum $\sigma(z)=f(z)$ exists and is uniform. 
Remark. In Theorem 7 the set $V$ may be replaced by a smaller one. To see this it suffices to set $\tilde{z}=\varrho_{1} z$ and apply Theorem 7. Thus, $V$ may be replaced by the $Q$-affine space $\widetilde{V}_{1}=\left\langle 1, \theta_{2}-\theta_{1}, \ldots, \theta_{M}-\theta_{1}\right\rangle_{Q}-\theta_{1}$.

Example. As an illustration of the previous results we consider the following example:

$$
f(z)=\omega(z)=[1 /(1-z)]+[1 /(1-\varrho z)] \text {, where } \varrho=e^{i 2 \pi \theta} \text { with } \theta \notin Q .
$$

Let $z=e^{i 2 \pi x}$ with $x \notin\langle 1, \theta\rangle_{Q}$. Then $m=1$ and

$$
g_{z}^{0}\left(z_{1}, z_{0}\right)=\left\{[1 /(z-1)]+[1 /(\varrho z-1)] z_{1}\right\} z_{0} .
$$

The limit set $L(z)$ is the closed annulus with center $\omega(z)=[1 /(1-z)]+$ $[1 /(1-\varrho z)]$, small radius || $1 /(z-1)|-| 1 /(\varrho z-1)||=r_{1}$ and great radius $|1 /(z-1)|+|1 /(1-\varrho z)|=r_{2}$. We set $|1 /(z-1)|=a>0$ and $|1 /(\varrho z-1)|=$ $b>0$ and write for the position vector $\omega(z)+r e^{i t}, r_{1} \leq r \leq r_{2}, 0 \leq t \leq 2 \pi$. Then for the measure $\mu_{z}$, we find

$$
\begin{array}{r}
d \mu_{z}=c r d r d t / \sqrt{(a+b+r)(a+b-r)(a-b+r)(-a+b+r)} \\
\text { where } c=1 / \pi^{2} .
\end{array}
$$

Remark. Under the assumptions of Theorem 7, one can show that, for every $z$ in $\mathbf{T}$ except the poles of $f$, the sequence of partial sums $S_{N}(z)=\sum_{0}^{N} a_{n} z^{n}$, $N=0,1,2, \ldots$ is uniformly distributed with respect to some compactly supported in $\mathbb{C}$ probability measure $\mu_{z}$, which may not be invariant under rotations with center $\sigma(z)=f(z)$ in general. The proof uses Weyl's Uniform Distribution Theorem and is a modification of the proof of Lemma 6. Further, for every $z$ in $T$ except a finite set, we have $\mu_{z}(f(z))=0$. This implies that, for every $z$ in $T$ except a finite set, the angular distribution of $S_{N}(z)$, $N=0,1, \ldots$, around $\sigma(z)=f(z)$ exists but it is not uniform in general.

Remark. One can also derive the following.

Proposition. Let $f$ be a rational function regular at 0 and let $\sum_{0}^{\infty} a_{n} z^{n}$ be its Taylor development. We denote by $S_{N}(z)=\sum_{0}^{N} a_{n} z^{n}$ the partial sums. Let $w$ be a point in the plane; then the following are equivalent.

(a) The sequences $S_{N}(w), N=0,1,2, \ldots$, is uniformly distributed with respect to a probability measure $\mu_{w}$ with compact support in $\mathbb{C}$.

(b) The series $\sum_{0}^{\infty} a_{n} w^{n}$ is $(C, 1)$ summable to a finite sum.

(c) (i) $w$ is not a pole of $f$.

(ii) $f$ is regular in the disk $\{z \in \mathbb{C}:|z|<|w|\}$.

(iii) If $f$ has any poles on $\{z \in \mathbb{C}:|z|=|w|\}$, these poles are simple.

\section{ANGULAR Distribution}

Theorem 7 does not cover the case of a rational function $f$ regular in a neighbourhood of $\bar{D}$. In this case we have $S_{N}(z) \rightarrow f(z)$, as $N \rightarrow+\infty$, $z \in T$; therefore the distribution of the sequence $S_{N}(z), N=0,1, \ldots$, is the Dirac mass at $f(z)$. This does not give any information about the angular distribution of the sequence $S_{N}(z), N=0,1, \ldots$, around $\sigma(z)=f(z)$. However, we prove the following. 
Proposition 8. Let $f$ be a rational function regular in a neighbourhood of $\bar{D}$, which is not a polynomial. Let $S_{N}(z), N=0,1, \ldots$, be the sequence of partial sums of the Taylor development of $f$. Then there is a denumerable subset $\Omega$ of the unit circle $T$, such that the following holds.

For every $z$ in $T \backslash \Omega$, the angular distribution of the sequence $S_{N}(z), N=$ $0,1, \ldots$, around $f(z)$ exists and this angular distribution is uniform.

Proposition 8 is a special case of the following theorem, where $(C, 1)$ summability is not assumed.

Theorem 9. Let $f$ be a rational function regular at 0 , which is not a polynomial. Let $S_{N}(z)$ be the partial sums of the Taylor development of $f$. Then there is a set $\Omega$, which is a denumerable union of straight lines passing through 0 , such that the following holds.

For every $z$ in $C \backslash \Omega$, the angular distribution of the sequence $S_{N}(z), N=$ $0,1,2, \ldots$, around $f(z)$ exists and this angular distribution is uniform.

In order to prove Theorem 9 we use Lemma 6 . The following trivial fact will also be useful: If $K_{n}>0, n=1,2, \ldots$, then for every complex sequence $P_{n}$, $n=1,2, \ldots$, the sequences $P_{n}$ and $K_{n} P_{n}$ are equivalent as regards angular distribution; if one of them has angular distribution, then the other does also and the two angular distributions coincide.

Suppose $a_{n} \in \mathbb{C}, n=1,2, \ldots$, is a sequence with uniform angular distribution and $b_{n} \rightarrow 0$, as $n \rightarrow+\infty$; then we cannot in general determine the angular distribution of the sequence $a_{n}+b_{n}$. However the following holds:

Suppose $a_{n} \in \mathbb{C}, n=1,2, \ldots$, is a sequence uniformly distributed with respect to a compactly supported probability measure $\mu$. Suppose also that $\mu(\{0\})=0$; then, the angular distribution of $a_{n}, n=1,2, \ldots$, around 0 exists. Further, suppose $b_{n} \rightarrow 0$, as $n \rightarrow+\infty$. Then $a_{n}+b_{n}, n=1,2, \ldots$, is uniformly distributed with respect to $\mu$. It follows that the angular distribution of the sequence $a_{n}+b_{n}, n=1,2, \ldots$, around 0 exists and coincide with that of $a_{n}, n=1,2, \ldots$.

We also need the following lemma.

Lemma 10. For $m=1,2, \ldots$ we denote $f_{m}(w)-1 /(1-w)^{m}$.

Let $\sum_{n=0}^{\infty} a_{n}^{m} w^{n}$ be the Taylor development of the function $f_{m}$ and let $S_{N}^{m}(w)$ $=\sum_{n=0}^{N} a_{n}^{m} w^{n}$. Then we have:

(i) $\left[(1 / m) f_{m}\right]^{\prime}=f_{m+1}$ and $\left[(1 / m) S_{N+1}^{m}\right]^{\prime}=S_{N}^{m+1}$.

(ii) $S_{N}^{1}(w)=f_{1}(w)-w^{N+1} f_{1}(w)$.

(iii) For $m \geq 2$ we have

$$
S_{N}^{m}(w)=f_{m}(w)-\left(\begin{array}{c}
N+m \\
m-1
\end{array}\right)\left[w^{N+1} f_{1}(w)+\sum_{k=2}^{m} c_{k}^{m}(N) w^{N+k} f_{k}(w)\right],
$$

where $c_{k}^{m}(N) \rightarrow 0$, as $N \rightarrow+\infty$.

(iv) For $m \geq 1$ and $w \neq 1$ we have

$$
S_{N}^{m}(w)=f_{m}(w)-\left(\begin{array}{c}
N+m \\
m-1
\end{array}\right) w^{N+1}\left[f_{1}(w)+o(1)\right] \text {, as } N \rightarrow+\infty .
$$

Proof. Parts (i) and (ii) can easily be verified. Combination of (i) and (ii) implies easily

$$
S_{N}^{2}(w)=f_{2}(w)-(N+2)\left\{w^{N+1} f_{1}(w)+[1 /(N+2)] w^{N+2} f_{2}(w)\right\}
$$


This gives (iii) for $m=2$. We proceed by induction on $m$ and we assume that (iii) holds for $m$ to prove it for $m+1$.

We have

$$
\begin{aligned}
S_{N}^{m+1}(w)= & {\left[(1 / m) S_{N+1}^{n}(w)\right]^{\prime} } \\
= & {\left[(1 / m) f_{m}(w)\right]^{\prime}-\frac{1}{m}\left(\begin{array}{c}
N+1+m \\
m-1
\end{array}\right) } \\
& \times\left[w^{N+2} f_{1}(w)+\sum_{k=2}^{m} c_{k}^{m}(N+1) w^{N+1+k} f_{k}(w)\right]^{\prime} \\
= & f_{m+1}(w)-\frac{1}{m}\left(\begin{array}{c}
N+m+1 \\
m-1
\end{array}\right)(N+2) \\
& \times\left\{\left(w^{N+2} f_{1}(w)\right)^{\prime} /(N+2)\right. \\
& \left.\quad+\sum_{k=2}^{m}\left[c_{k}^{m}(N+1) /(N+2)\right] \cdot\left(w^{N+1+k} f_{k}(w)\right)^{\prime}\right\} .
\end{aligned}
$$

We observe that $(1 / m)\left(\begin{array}{c}N+1+m \\ m-1\end{array}\right) \cdot(N+2)=\left(\begin{array}{c}N+m+1 \\ m\end{array}\right)$, as required. Further

$$
\left(w^{N+2} f_{1}(w)\right)^{\prime} /(N+2)=-w^{N+1} f_{1}(w)+[1 /(N+2)] w^{N+2} f_{2}(w)
$$

and

$$
\begin{aligned}
{\left[c_{k}^{m}(N+1) /(N+2)\right]\left[w^{N+1+k} f_{k}(w)\right]^{\prime} } & \\
= & c_{k}^{m}(N+1) \cdot[(N+1+k) /(N+2)] f_{k}(w) \\
& +\left[c_{k}^{m}(N+1) k /(N+2)\right] w^{N+1+k} f_{k+1}(w) .
\end{aligned}
$$

Since $c_{k}^{m}(N+1) \rightarrow 0$, as $N \rightarrow+\infty$, we see that (iii) holds for $m+1$ also. This proves (iii).

Part (iv) follows immediately from parts (ii) and (iii). Q.E.D.

Now, we are ready to prove Theorem 9.

Proof of Theorem 9. Developing in partial sums we take

$$
f(z)=P(z)+\sum_{j=1}^{M} A_{j} /\left(1-\varrho_{j} z / R_{j}\right)^{m_{j}}, \text { where }\left|\varrho_{j}\right|=1, R_{j}>0,
$$

$m_{j}$ integer, $m_{j} \geq 1, A_{j} \neq 0, m_{j} \geq 1$, and $P$ is a polynomial. The vectors $\left(R_{j}, \varrho_{j}, m_{j}\right), j=1, \ldots, M$, are distinct. We set

$$
R=\min \left\{R_{j}: j=1, \ldots, M\right\}
$$

and

$$
m=\max \left\{m_{j}: j-1, \ldots, M, R_{j}=R\right\} .
$$

For $z$ different from 0 and the poles of $f$, we write $z=|z| \varrho,|z|>0,|\varrho|=1$, and we consider the partial sum $S_{N}(z)$ of the Taylor development of $f$. For $N \geq \operatorname{deg} P$, using Lemma 10 we obtain the following.

$$
S_{N}(z)-f(z)=\sum_{j=1}^{M}\left(\varrho_{j}|z| \varrho / R_{j}\right)^{N+1}\left(\begin{array}{l}
N+m_{j} \\
m_{j}-1
\end{array}\right)\left\{\left[A_{j} /\left(-1+\varrho_{j} z / R_{j}\right)\right]+o(1)\right\}=
$$
I + II + III , where 
I is the sum on $\left\{j=1, \ldots, M: R_{j}=R, m_{j}=m\right\}$, II is the sum on $\left\{j=1, \ldots, M: R_{j}=R, m_{j}<m\right\}$, III is the sum on $\left\{j=1, \ldots, M: R_{j}>R\right\}$.

We consider the resulting formula for $\left[S_{N}(z)-f(z)\right](R /|z|)^{N+1} \cdot 1 /\left(\begin{array}{c}N+m \\ m-1\end{array}\right)$. Term I gives

$$
\left[\sum_{\substack{R_{j}=R \\ m_{j}=m}} A_{j} \varrho_{j}^{N+1} /\left(-1+\varrho_{j} z / R\right)\right] \varrho^{N+1}+o(1) \text {, since }\left|\varrho_{j}\right|=|\varrho|=1 .
$$

Term II gives

$$
\sum_{\substack{R_{j}=R \\
m_{j}<m}}\left(\varrho_{j} \varrho\right)^{N+1}\left(\begin{array}{c}
N+m_{j} \\
m_{j}-1
\end{array}\right)\left(\begin{array}{c}
N+m \\
m-1
\end{array}\right)\left\{\left[A_{j} /\left(-1+\varrho_{j} z / R_{j}\right)\right]+o(1)\right\}=o(1),
$$

because we have $\left|\varrho_{j}\right|=|\varrho|=1$ and $\left(\begin{array}{c}N+m_{j} \\ m_{j}-1\end{array}\right) /\left(\begin{array}{c}N+m \\ m-1\end{array}\right)$ grows as $N^{\left(m_{j}-m\right)}, m_{j}<m$. Term III also gives

$$
\begin{gathered}
\sum_{R_{j}>R}\left(\varrho_{j} \varrho\right)^{N+1}\left(R / R_{j}\right)^{N+1}\left(\begin{array}{c}
N+m_{j} \\
m_{j}-1
\end{array}\right) /\left(\begin{array}{c}
N+M \\
m-1
\end{array}\right)\left\{\left[A_{j} /\left(-1+\varrho_{j} z / R_{j}\right)\right]+o(1)\right\} \\
=o(1),
\end{gathered}
$$

because $\left|\varrho_{j}\right|=|\varrho|=1,0<R / R_{j}<1$ and $\left(\begin{array}{c}N+m_{j} \\ m_{j}-1\end{array}\right) /\left(\begin{array}{c}N+m \\ m-1\end{array}\right)$ grows as $N^{\left(m_{j}-m\right)}$.

Thus we find $\left[S_{N}(z)-f(z)\right](R /|z|)^{N+1} 1 /\left(\begin{array}{c}N+m \\ m-1\end{array}\right)=\delta_{N+1}(\varrho, z / r)+o(1)$, where following the notation of Lemma 6 we have

$$
\delta_{N}(\varrho, z / R)=\left\{\sum_{\substack{R_{j}=R \\ m_{j}=m}}\left[A_{j} \varrho_{j}^{N} /\left(-1+\varrho_{j} z / R\right)\right]\right\} \varrho^{N} .
$$

We notice that the $\varrho_{j}$ 's entering in the sum are distinct and therefore, Lemma 6 applies. It follows that there are a denumerable subset $\Gamma \subset \mathbf{T}$ and a finite set $B \subset \mathbb{C}$, such that for $\varrho=z /|z| \in T \backslash \Gamma$ and $z / R \in \mathbb{C} \backslash B$ the following holds:

The sequence $\delta_{N}(\varrho, z / R), N=0,1, \ldots$, is uniformly distributed with respect to a compactly supported in $\mathbb{C}$ probability measure $\lambda_{\varrho, z / R} \equiv \mu_{z}$. The measure $\mu_{z}$ is invariant under rotations around 0 and $\mu_{z}(\{0\})=0$.

It follows that for $z$ in $\mathbb{C} \backslash\{0\}$ with $z / R \notin B$ and $z /|z| \notin \Gamma$ the sequence

$$
\left[S_{N}(z)-f(z)\right](R /|z|)^{N+1} 1 /\left(\begin{array}{c}
N+m \\
m-1
\end{array}\right)=\delta_{N+1}(z /|z|, z / R)+o(1)
$$

is also uniformly distributed with respect to $\mu_{z}$. By the invariance of $\mu_{z}$ under rotations around 0 and the fact that $\mu_{z}(\{0\})=0$, it follows that the angular distribution exists and is uniform. We set $\Omega$ to be the union of all straight lines through 0 and the points of $B \cup \Gamma$ and the poles of $f$ and we obtain the result. Q.E.D.

Remark. One can also prove that under the hypothesis of Theorem 9, for every $z$ in $\mathbb{C}$ except a finite set, the angular distribution of the sequence $S_{N}(z)$, $N=0,1,2, \ldots$, around $f(z)$ exists, but it is not in general uniform. 
Example. Let us consider the simple example $f(z)=1 /[1-z]$. For $z \neq 1$ the partial sum $S_{N-1}(z)$ of the Taylor development is $S_{N-1}(z)=f(z)+z^{N} / z-1$. Set $z=|z| e^{i 2 \pi \theta}$; then $S_{N-1}(z)-f(z)=\left[|z|^{N} /(z-1)\right] e^{i 2 \pi \theta N}$. We see that, for all $z \neq 1,0$ the angular distribution exists. Furthermore, if $\theta \notin Q$, then this angular distribution is uniform.

\section{REFERENCES}

1. G. H. Hardy and E. M. Wright, An introduction to the theory of numbers, 5th ed., Oxford at the Clarendon Press, 1979.

2. J.-P. Kahane, Sur la structure circulaire des ensembles de points limites des sommes partielles d'une série de Taylor, Acta Sci. Math. (Szeged) 45 (1983), 247-251.

3. E. S. Katsoprinakis, On a theorem of Marcinkiewicz and Zygmund for Taylor series, Ark. for Mat. 27 (1989), 105-126.

4. E. S. Katsoprinakis and V. Nestoridis, Partial sums of Taylor series on a circle, Ann. Inst. Fourier 39 (1989), 715-736.

5. E. S. Katsoprinakis, Taylor series with limit-points on a finite number of circles, Trans. Amer. Math. Soc. 337 (1993), 437-450.

6. E. S. Katsoprinakis and V. Nestoridis, An application of Kronecker's Theorem to rational functions, Math. Ann. 298 (1994), 145-166.

7. L. Kuipers and H. Niederreiter, Uniform distribution of sequences, Wiley-Interscience, 1974.

8. J. Marcinkiewicz and A. Zygmund, On the behavior of trigonometric series and power series, Trans. Amer. Math. Soc. 50 (1941), 407-453.

9. V. Nestoridis and S. K. Pichorides, The circular structure of the set of limit points of partial sums and Taylor series, Séminaire d'Analyse Harmonique, Année 1989-90, Université de Paris-Sud, Mathématiques, Bâtiment 425, 91405 Orsay, Cedex, France, pp. 71-77.

10. V. Nestoridis, Limit points of partial sums of Taylor series, Matematika 38 (1991), 239-249.

11. L Limit set of exponential sums, in preparation.

12. _ Limit points of partial sums of Taylor series on a circle, Proc. Complex Analysis and Applications' 91 with Symposium on Generalized Functions (Varna, September 15-21, 1991) (to appear).

13. A. Zygmund, Trigonometric series, 2nd ed., Vols. I, II, reprinted, Cambridge Univ. Press, 1979.

Department of Mathematics, University of Crete, Iraklion, Crete, Greece 